\title{
Investigation of Transmit and Receive Characteristics of Laboratory Model for the Parametric Echosounder
}

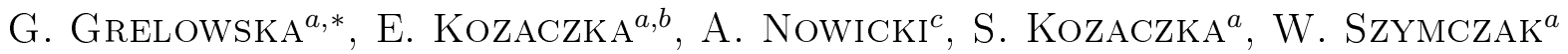 \\ AND R. TYMKIEWICZ ${ }^{c}$ \\ ${ }^{a}$ Polish Naval Academy, Smidowicza 69, 81-103 Gdynia, Poland \\ ${ }^{b}$ Gdansk University of Technology, Narutowicza 11/12, 80-233 Gdańsk, Poland \\ ${ }^{c}$ Institute of Fundamental Technological Research, Polish Academy of Sciences, Pawinskiego 5B, 02-106 Warszawa, \\ Poland
}

\begin{abstract}
Investigation and operation of devices based on nonlinear interaction of high-intensity waves have been carried out for several decades. However, it is still a new tool to study the properties of the water environment and especially the upper layers of the seabed. This paper describes the results of tests of the new device that is intended to be applied for sounding underwater areas. The device has been designed and built in collaboration with the Department of Ultrasound of the Institute of Fundamental Scientific Researches of the Polish Academy of Sciences in Warsaw.
\end{abstract}

DOI: 10.12693/APhysPolA.123.1094

PACS: 43.30.Ma, 43.30.Pc, 43.25.Jh

\section{Introduction}

The use of devices to sound the seabed called subbottom profilers provides opportunities for acoustic waves penetration at a depth of tens of meters into the bottom [1-4]. Such possibilities are represented only by the waves with a relatively low frequency up to a maximum of $20 \mathrm{kHz}$. Because using conventional methods to produce waves with frequency of a few $\mathrm{kHz}$ requires a very large transducer with a large capacity power transmission system as well as due to the poor directivity of the transmitting antenna, the traditional solutions seem to be technically inefficient [5].

This technological gap is filled by devices based on the parametric generation of waves theoretically initiated by Westerwelt [6], Khokhlow, Zakolotska [7] and Kuzniecov [8]. It has been proven [9] that in the area of propagation of two collinear acoustic beams modules of wave vectors of which are slightly different there is the difference frequency wave formed characterized by the angular frequency

$$
\Delta \omega=\omega_{1}-\omega_{2},
$$

where $\omega_{1}, \omega_{2}$ are angular frequencies of primary waves, that has almost the same features as the so-called primary waves, i.e. high directivity and spatial resolution [10]. Because source of the wave is a finite volume environment (area of mutual impact of the primary waves) rather than the surface, beam pattern of the wave does not have side lobes [11].

The theory of differential wave parametric generation is complex, and in this particular case it does not seem

\footnotetext{
*corresponding author; e-mail: g.grelowska@amw.gdynia.pl
}

necessary to be quoted. Therefore to describe the amplitude of the wave frequency of which is equal to difference and sum of frequencies of primary waves, there is given only the relation as a result of solutions of Burgers equation of the form [9] (valid for $\omega_{1}>\omega_{2}$ ):

$$
\begin{gathered}
p_{2}(x, \tau)=\frac{\varepsilon p_{01} p_{02}\left(\omega_{1}-\omega_{2}\right)}{2 b \omega_{1} \omega_{2}}\left[\exp \left[\frac{b\left(\omega_{1}^{2}+\omega_{2}^{2}\right)}{2 \rho_{0} c_{0}^{3}} x\right]\right. \\
\left.-\exp \left[\frac{b\left(\omega_{1}-\omega_{2}\right)^{2}}{2 \rho_{0} c_{0}^{3}} x\right]\right] * \sin \left(\omega_{1}-\omega_{2}\right) \tau,
\end{gathered}
$$

where $x$ is the axis perpendicular to the entire radiating area, $p_{01}$ is the amplitude of the primary wave with angular frequency $\omega_{1}, p_{02}$ is the amplitude of the primary wave of angular frequency $\omega_{2}, \rho_{0}$ is the density of the undisturbed environment, $c_{0}$ is the wave velocity in the undisturbed environment, $\varepsilon$ is the nonlinear coefficient, a dimensionless value, for water equaling about 3.5, and $\tau$ is the time.

It should be emphasized while analyzing the relation (2) that for case $\omega_{1} \rightarrow \omega_{2}$, the value $p_{2}(x, \tau) \rightarrow 0$. This is an important limitation of use the parametric generation of acoustic waves.

Results of comparison of the changes in the amplitude of the primary wave and differential wave (for plane waves) [11] are shown in Fig. 1.

\section{Measuring set up}

The new parametric antenna has been investigated in order to provide its operation characteristics. The first point of the study was to measure the directional characteristics of both the primary and differential frequency waves. The measurements were carried out at the Technical University of Gdańsk where a 30-meter long, 3-meter wide and 1.8-meter deep water tank is located. Main 


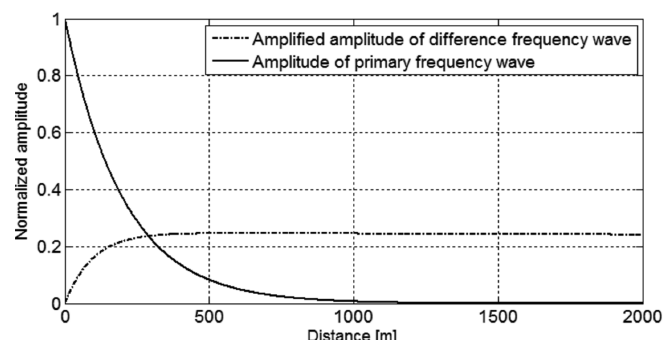

Fig. 1. Changes in the normalized amplitude of one of the primary waves and the wave of frequency equal to the difference of frequencies of primary waves as a function of distance calculated for a plane wave.

beam was directed horizontally along the longest dimension of the water tank, because in order to form the differential frequency wave by means of the parametric effect a certain distance from the radiating primary waves surface is needed. Because the both depth and the width of the tank were limited, the first important step was to mount and direct correctly the main lobe of transmitting array. During measurement the antenna was fixed to a stationary mount. The movable element was a platform on rails with fixed 3D positioning system called "ISEL" with the receiving hydrophone type 8104 Bruel\&Kjaer mounted to the one of the stage of the positioning system (that gave opportunity to locate sensor with the accuracy of 25 micrometers). The hydrophone move, controlled by a computer, allowed for performing all the moves necessary to determine the transmitting characteristics of the antenna.

\section{Research station}

Figure 2 presents the arrangement scheme of the elements for measurements in the water tank. In the side opposite to the antenna there was a damping matrix placed to minimize echo from the concrete wall.

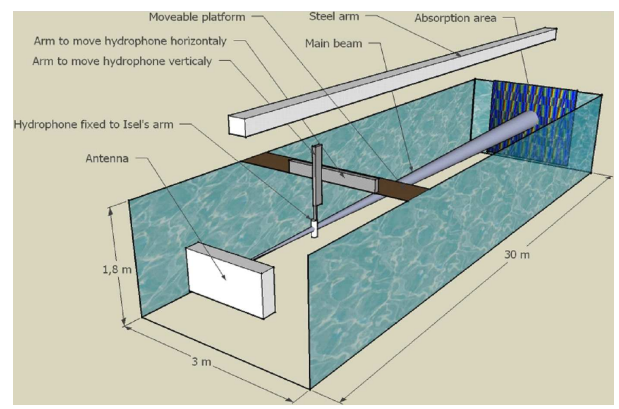

Fig. 2. The station used to measure the antenna's directivity.

The station to measure directivity characteristics (Fig. 3) can be divided into two main blocks: (a) the antenna mount and (b) the measurement section.

a) The antenna mount assures:
- Adjustment of depth of immersion of the antenna to set it in the middle of the depth. Such facility allows for elimination of reverberations coming from water tank bottom and surface of water. Changing depth was realized by blocking sliding element on the proper level with accuracy of one millimeter.

- Control of horizontal and vertical direction of main lobe transmitting. Due to small values of angles which should be set correctly during rotating of transmitting element, it was necessary to build a precise positioning mount. Fig. 4 shows a photo of transducer array fixed to the special mount made of three rectangular profiles with threaded rods inside. The arms allowed for the use of less power during positioning and made the whole process faster and more effective. Adjustment can be done by screwing the special positioning knobs at ends of three control arms above the water (two of them are responsible for horizontal control and one for vertical).

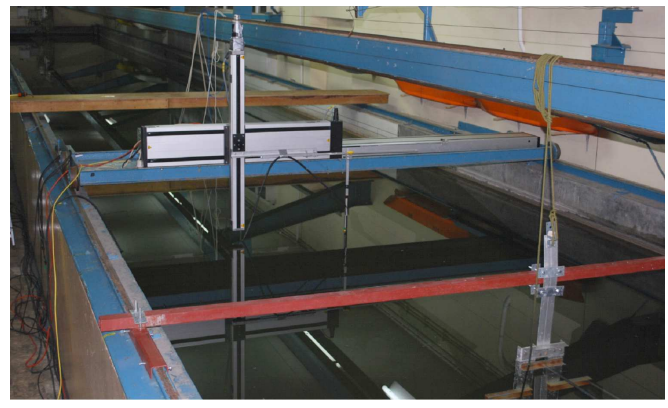

Fig. 3. Photo of ISEL and antenna mounting.

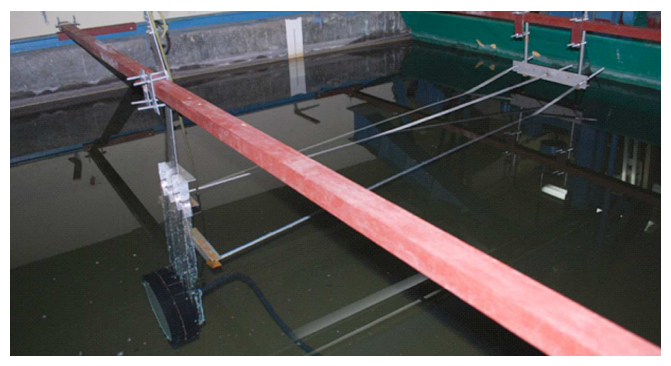

Fig. 4. Antenna mount for precise beam positioning.

b) The measurement section

For measurements, Tektronix oscilloscope with four input channels and the external trigger was used as the main signal receiving unit. It was additionally connected via ethernet connection to notebook where selected data were stored on disc or measured values stored in the tables. The trigger of the generator was connected directly to external input of Tektronix oscilloscope. It was necessary for proper assessment of whether the correct pulse is measured. The used Agilent generator allowed to 
generate two synchronized pulses with different primary frequencies that were supplied to six separated amplifiers powering six transmitting sections (each consisting of three piezoceramic elements) of transducers in the antenna.

Three different signals were possible to be displayed on the measuring device:

a) signal from hydrophone $8104 \mathrm{~B} \& \mathrm{~K}$ mounted on " ISEL" stage,

b) signal from receiving transducer placed in the center of the parametric antenna,

c) signal supplied to the antenna's amplifier.

In order to observe changes of differential sounding pulse, it was necessary to filter signal from hydrophone or receiving transducer built in the parametric antenna. Filtering was realized with the use of the band pass filter. Later, when antenna will be used in natural sea condition $[12,13]$ it should be processed to get an adequate image of the structure of the seabed [14-16]. The same notebook, which was working with osciloscope, was also used to send commands to the positioning system "ISEL". Figure 5 shows the schematic diagram of connections between sensors and measurement units.

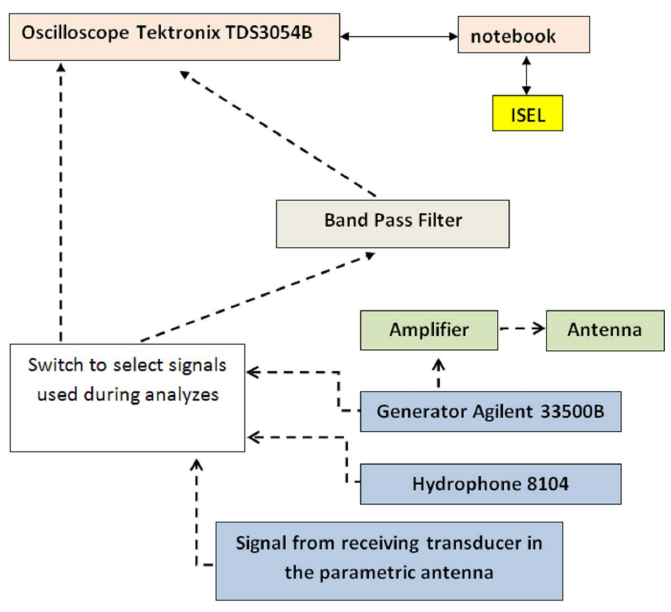

Fig. 5. Schematic diagram of connections.

The quality of the data obtained from both the receiving transducer in the antenna and the receiving hydrophone allowed for the complete and correct realization of the steps in the study plan.

\section{Results}

Some characteristics obtained during the research are shown in the graphs below. Examples were obtained during measurements in horizontal and vertical plane and in two parametric antenna settings:

1. Individual sections of transmitting antenna sent pulses with frequencies of $147 \mathrm{kHz}$ and $155 \mathrm{kHz}$, respectively. Directivity patterns of primary waves and the differential wave are presented in Fig. 6 and Fig. 7.

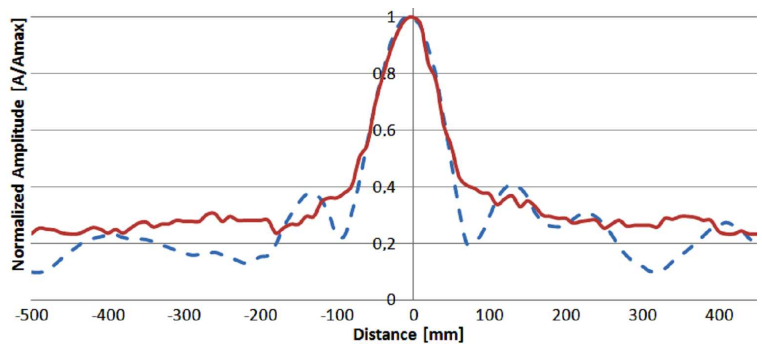

Fig. 6. Horizontal directivity characteristics of primary (dotted line $147 \mathrm{kHz}$ ) and secondary (solid line $8 \mathrm{kHz}$ ) waves at the plane distant $2 \mathrm{~m}$ from antenna.

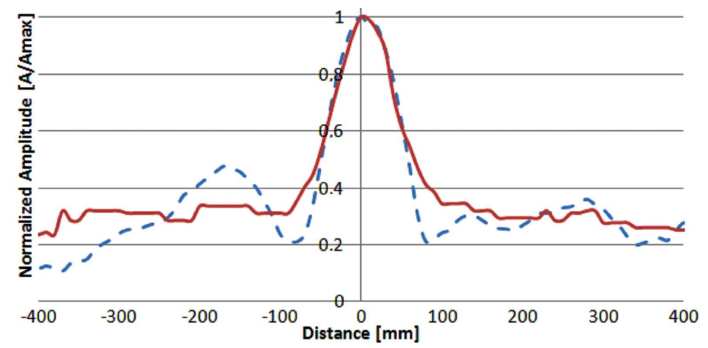

Fig. 7. Vertical directivity characteristics of primary (dotted line $147 \mathrm{kHz}$ ) and secondary (solid line $8 \mathrm{kHz}$ ) waves at the plane distant $2 \mathrm{~m}$ from antenna.

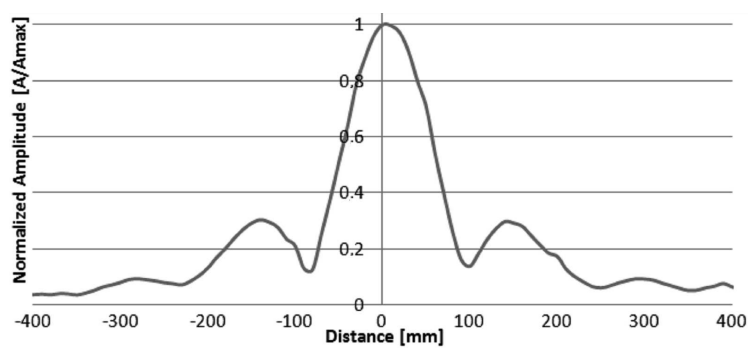

Fig. 8. Horizontal directivity characteristics determined for one of the primary waves with frequency of $147 \mathrm{kHz}$ transmitted by all sections of transducers.

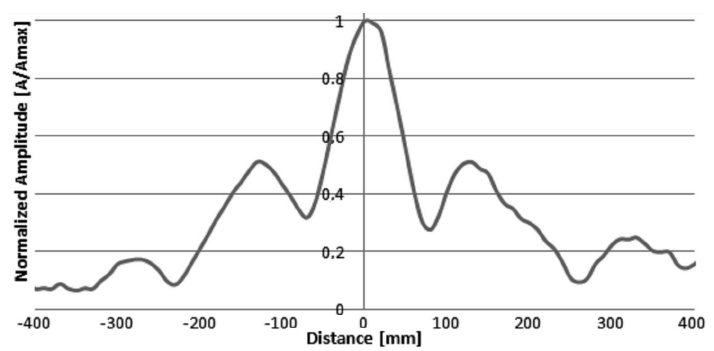

Fig. 9. Vertical directivity characteristics determined for one of the primary waves with frequency of $147 \mathrm{kHz}$ transmitted by all sections of transducers. 
2. All the sections of transducers were supplied with pulses with the frequency of $147 \mathrm{kHz}$. The beam patterns of the primary wave in vertical as well as horizontal plane are shown in Fig. 8 and Fig. 9.

\section{Conclusions}

Laboratory research of the transmitting antenna which was the main element of the parametric source provided the basic information about characteristics of acoustic waves radiated by the antenna. The results confirm the theoretically predicted parametric source properties, such as the generation of low frequency waves only within the primary wave beam, resulting in a narrow directional radiation characteristics without side lobes.

The obtained data allow for estimating the spatial resolution of parametric source and assessing of the efficiency of energy transfer from primary waves to the secondary one.

Additional results confirm correct preparation of the measuring set up that allowed for obtaining the presented directivity characteristics during the research.

\section{Acknowledgments}

The investigation was partially supported by the Ministry of Science and Higher Education in the framework of the fund for statutory activities of the Polish Naval Academy.

\section{References}

[1] G. Grelowska, E. Kozaczka, Acta Physica Polonica A 118, 66 (2010).

[2] P. Blondel, A. Caiti, Buried Waste in the Seabed Acoustic Imaging and Bio-Toxicity, University of Bath, Bath 2007.
[3] E. Kozaczka, G. Grelowska, S. Kozaczka, W. Szymczak, Archiv. Acoust. 38, 99 (2013).

[4] E. Kozaczka, G. Grelowska, S. Kozaczka, Acta Physica Polonica A 118, 91 (2010).

[5] K. Naugolnykh, L. Ostrovsky, Nonlinear Wave Processes in Acoustics, Cambridge University, Cambridge 1998.

[6] P.J. Westervelt, J. Acoust. Soc. Am. 35, 535 (1953).

[7] E.A. Zabolotskaya, R.V. Khokhlov, Sov. Phys. Acoust. 15, 35 (1969).

[8] V.P. Kuznetsov, Ak. Zh. 16, 548 (1970).

[9] E. Kozaczka, Introduction to the theory of nonlinear hydroacoustics, Polish Naval Academy Press, Gdynia 1987, (in Polish).

[10] G. Grelowska, Elastic waves of finite amplitude in water, Polish Naval Academy Press, Gdynia 2001, (in Polish).

[11] M.F. Hamilton, D.T. Blackstock, Nonlinear acoustics, Academic Press, 1998.

[12] G. Grelowska, Archiv. Acoust. 25, 359 (2000).

[13] G. Grelowska, E. Kozaczka, Nonlinear properties of sea water, Polish Naval Academy Press, Gdynia 1996, (in Polish).

[14] G. Grelowska, E. Kozaczka, S. Kozaczka, W. Szymczak, Polish Maritime Research 19, (4)3 (2012).

[15] D. Walter, D. Lambert, D. Young, K. Stephens, GeoMarine Lett. 17, 260 (1997).

[16] J. Wunderlich, S. Müller, Int. Ocean Syst. 7, (4)6 (2003). 